\title{
THE ELECTROSTATIC BREAKDOWN ON A METAL-DIELECTRIC JUNCTION IMMERSED IN A PLASMA
}

\author{
Boris V. Vayner*, Joel T. Galofaro, and Dale C. Ferguson \\ NASA/Glenn Research Center, Cleveland, Ohio 44135, USA
}

\begin{abstract}
New results are presented of an experimental study and theoretical analysis of arcing on metal-dielectric junctions immersed in low-density plasmas. Two samples of conventional solar arrays and four different metal-quartz junctions have been used to investigate the effects of arcing within a wide range of neutral gas pressures, ion currents, and electron number densities. The effect of surface conditioning (decrease of arc rate due to outgassing) was clearly demonstrated. Moreover, a considerable increase in arc rate due to absorption of molecules from atmospheric air has been confirmed. It has been proved that the arc inception mechanism in plasma is different from one in vacuum.
\end{abstract}

\section{INTRODUCTION}

The current paper is a continuation of our study [1-3] of arc inception on a triplejunction with a conductor negatively biased with respect to the surrounding plasma. Previous experiments have clearly demonstrated that an electrostatic discharge occurs when the electric field strength is approximately $1 \mathrm{MV} / \mathrm{m}$, which is almost one order of magnitude lower than is needed for initiation of a vacuum arc [4]. In order to explain this fact we suggested that the ionization of adsorbed gas (water vapor) plays the main role in the generation of initial plasma cloud and the plasma sheath which lead to the enhancement of an electric field. Two field enhancement mechanisms, protrusions on a metal surface and plasma

"Ohio Aerospace Institute, Cleveland, OH 44142, USA polarization, can provide a high enough electric field $(1-2 \mathrm{GV} / \mathrm{m})$ to generate cold emission current with the density $10^{11}-10^{12}$ $\mathrm{A} / \mathrm{m}^{2}$. The plasma conductivity allows an electric current of 10-50 A, depending on the conditions of the experiments, to discharge a negatively biased electrode for short time intervals (10-50 $\mu \mathrm{s})$.

A simple model of a discharge quenching due to water ions dissociative recombination has been developed, and its prediction of the relation between pulse width and capacitance $\left(\tau \propto C^{l / 2}\right)$ is confirmed for a wide range of parameters $(C=0.01-1 \mu \mathrm{F}, U=300-800 \mathrm{~V})$. Moreover, hydrogen and hydroxyl spectral lines were observed in emission, and measurements revealed strong correlations between the intensity of $\mathrm{H}_{\alpha}$ line and the value of the initial electrical charge. The observation of metal $(\mathrm{Ag}, \mathrm{Cu})$ spectral lines is an unarguable argument in favor of a high density Fowler-Nordheim current heating a metallic protrusion to very high temperatures. Together with the observed decrease of arc rate with number of arcs (conditioning) and a considerable rise of arc threshold for a sample that has been kept under high vacuum for a few days, all these details can be incorporated in a cohesive model of arc inception.

The analysis of optical spectra (240$800 \mathrm{~nm}$ ) reveals intense narrow atomic lines $(\mathrm{Ag}, \mathrm{Cu}, \mathrm{H})$ and wide molecular bands $(\mathrm{OH}, \mathrm{CH}, \mathrm{SiH}, \mathrm{SiN})$ that confirm a complicated mechanism of arc plasma generation. The rate of plasma contamination due to arcing was measured by employing a mass-spectrometer. These 
measurements provided quite reliable data for the development of a theoretical model of plasma contamination. In conclusion, the arc threshold for the solar array sample was increased to above $350 \mathrm{~V}$ (from 190 V) by keeping sample in vacuum (20 $\mu$ Torr) for seven days. The results obtained are important for the understanding of the arc inception mechanism, which is absolutely essential for progress toward the design of highvoltage solar arrays for space applications.

\section{EXPERIMENTAL SETUP}

All our experiments were performed in the large vacuum chamber and small bell jar installed in the Plasma Interaction Facility (PIF) at NASA's Glenn Research Center. The vacuum equipment provides pressure as low as $10^{-7}$ Torr. Two Penning sources and a hollow cathode are installed to generate argon and xenon plasmas with electron density $n_{e}=(0.1-10) \cdot 10^{5} \mathrm{~cm}^{-3}$, with temperature $T_{e}=0.3-1.2 \mathrm{eV}$, and neutral gas pressure $p=(0.3-7) \cdot 10^{-5}$ Torr. These conditions can be kept steady during the experiment. The sample or set of samples (Fig.1) is vertically mounted in the middle of the chamber. The sample is biased to a voltage power supply through a capacitor and a $10 \mathrm{k} \Omega$ resistor network back to ground. Diagnostic equipment includes one spherical Langmuir probe with diameter $\mathrm{d}=2 \mathrm{~cm}$, one current probe to measure discharge current, and one voltage probe that allows us to register voltage pulse on the sample during the discharge. To allow us to measure optical and mass spectra and to simulate real capacitance of the device an additional capacitor $\mathrm{C}=1-2 \mu \mathrm{F}$ is installed between the sample and ground (Fig.2). To find the most probable arcing sites we install a video camera and VCR. To determine the species that provide ions due to cathode evaporation and/or outgassing we install an optical spectrometer with a CCD camera that provides spectral resolution better than $0.5 \mathrm{~nm}$ within the range 200$850 \mathrm{~nm}$, and a quadruple massspectrometer.
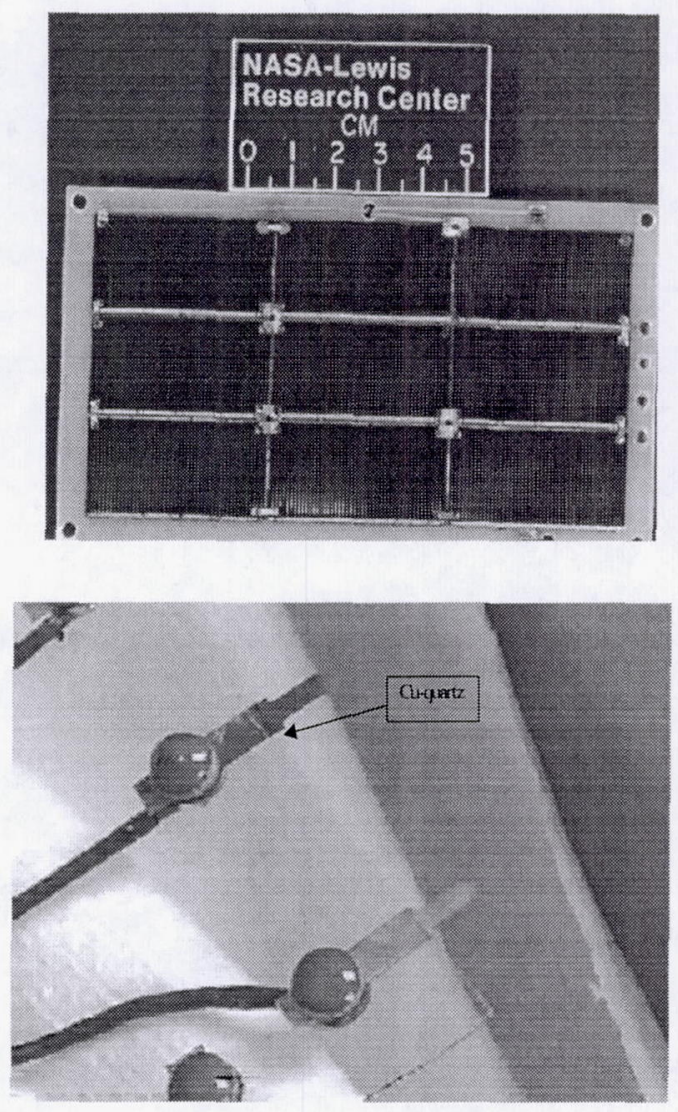

Fig.1. Solar array sample and quartzmetal junctions are used to study arc inception mechanism.

\section{EXPERIMENTAL RESULTS}

Examples of arc spectra are shown in Fig.3. Both a hydrogen line $\left(\mathrm{H}_{\alpha}\right)$ and a hydroxyl band were observed in emission for arcs on the solar array sample and the quartz-copper junction. Moreover, their relative intensities are practically equal $\frac{I\left(H_{\alpha}\right)}{I(O H)} \approx 4$ if the most intensive line 306 $\mathrm{nm}$ is used as a characteristic of the 
hydroxyl band intensity. This near equality is a very strong argument in favor of water desorption and an ionization mechanism for electrostatic discharge inception.

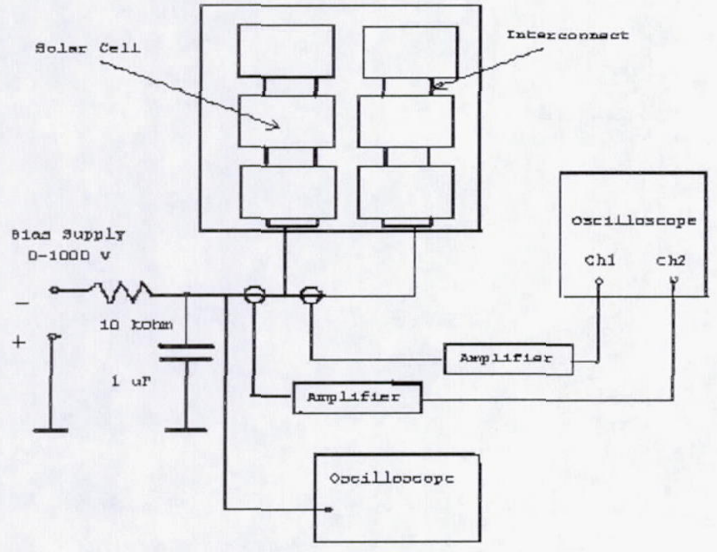

Fig.2. Additional capacitor is installed between negatively biased electrode and ground.

Atomic metal lines were also observed with high spectral intensity. These lines are easily identified: $324.8 \& 327.4 \mathrm{~nm}$ copper lines, and 328.1 \& $338.3 \mathrm{~nm}$ silver lines. According to NIST data [5] the transition probabilities for $\mathrm{Ag}$ and $\mathrm{Cu}$ are similar and $A \approx 1.4 \cdot 10^{8} \mathrm{~s}^{-1}$, while the transition probability for hydrogen is considerably less: $A_{32} \approx 0.7 \cdot 10^{8} \mathrm{~s}^{-1}$. The ratio of number densities of excited atoms can be estimated as

$$
X=\frac{N^{*}(H)}{N^{*}(M)}=\frac{I(656)}{I(328)} \cdot \frac{\alpha(328) \cdot A}{\alpha(656) \cdot A_{32}}
$$

where $N^{*}(M)$ is the number density of excited metal atoms ( $\mathrm{Cu}$ or $\mathrm{Ag}), I(\lambda)$ is the observed line intensity, and $\alpha(\lambda)$ is the photocathode quantum efficiency.

Substituting measured intensities in Eq.(1) results in the following estimate: $X=0.6-0.7$. One also has to take into account the possibility of electron impact excitation of metal lines (energy level $\sim 3.5 \mathrm{eV}$ ) that may greatly increase this estimate (1). It does not seem possible to determine the relative densities of metal and hydrogen ions without a computer simulation of all relative processes in the arc plasma.
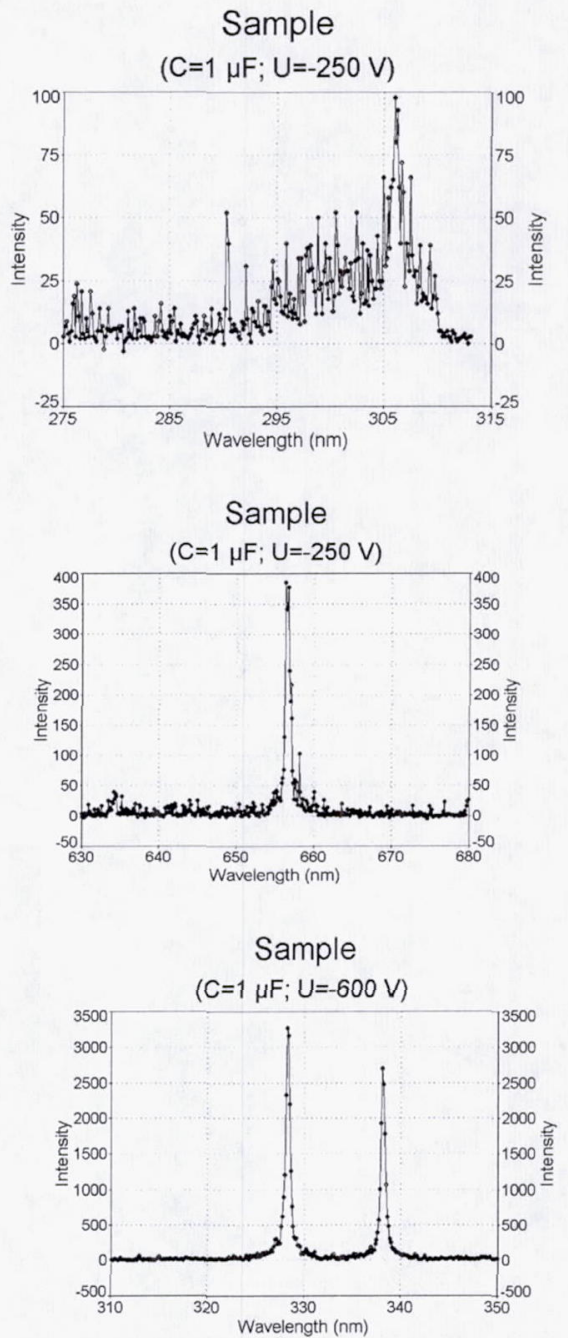

Fig.3. Arc spectra demonstrate the presence of hydroxyl, hydrogen, and silver in the arc plasma.

Halpha Intensity vs.charge $y=a+b x^{2}$

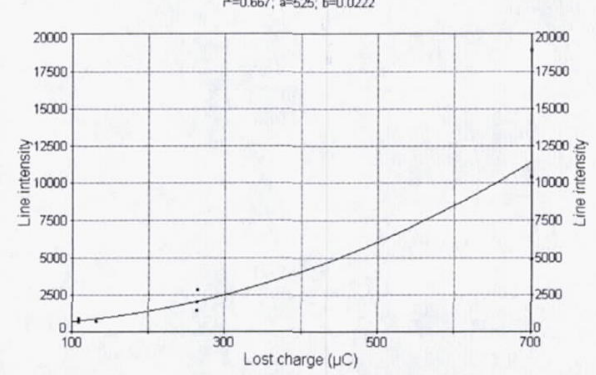

Fig.4. The intensity of $H_{\alpha}$ spectral line depends on bias voltage and capacitance rather strongly. 
Two more observations support the hypothesis of desorbed water ionization as the main source of the arc development: a) one triple junction can experience 15-20 ares being biased at fixed voltage when the capacitance is low $(C=0.01 \mu \mathrm{F})$, but the same junction arcs only 3-5 times with bigger capacitance $(C=1 \mu \mathrm{F})$; b) the intensity of the hydrogen spectral line $\left(H_{\alpha}\right)$ increases with increasing lost electrical charge (Fig.4).

The rate of the background plasma contamination caused by arcing was also measured by employing the quadruple mass spectrometer. Several examples of mass spectra are shown in Fig.5. Simple analytical model [6] confirms the observation that a real chance to register an increase in partial pressure exists for species with background partial pressure lower than $2.5 \mu$ Torr. The measured increase of hydrogen partial pressure (Fig.6) is in a quite good agreement with the analytical model, and this result can be considered as one more argument in favor

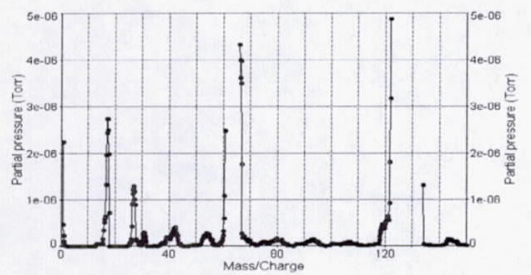

a)

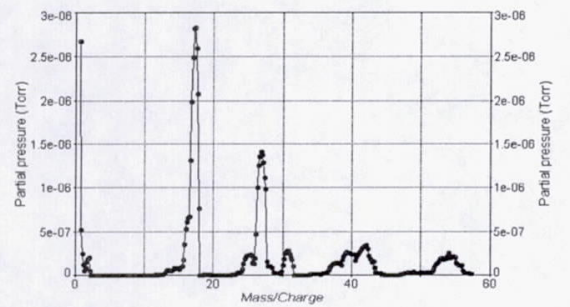

b)

Fig.5. Examples of mass spectra: a)xenon plasma with $\mathrm{n}_{\mathrm{e}}=10^{6} \mathrm{~cm}^{-3}$; b)arcing on the sample with rate $1 \mathrm{arc} / \mathrm{s}$. of the arc inception model developed in Ref.7.

It should be stressed that discharge cannot be initiated in vacuum between two metallic electrodes separated by a quartz plate even under maximum field strength achieved in this experiment $(1.5 \mathrm{MV} / \mathrm{m})$.

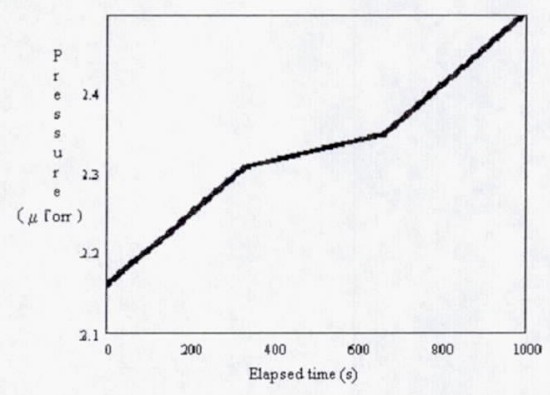

Fig.6. Change in partial pressure is shown for hydrogen atoms.

\section{REFERENCES}

[1]. Vayner, B.V., Ferguson, D.C., and Galofaro, J.T. XVIII International Symp. on Discharges and Electrical Insulation in Vacuum, August 1721, 1998, Eindhoven, Netherlands, Vol. 2, pp.824- 827

[2]. Vayner, B.V., Ferguson, D.C., Galofaro, J.T., and Snyder, D.B. $40^{\text {th }}$ Annual Meeting, APS Division of Plasma Physics, November 16-20, 1998, New Orleans, LA, USA (Bull. Of the American Phys. Soc., Vol.43, No.8, p.1728)

[3]. Vayner, B., Galofaro, J., Ferguson, D., and Degroot, W. Proc. XXVth International Conference on Phenomena in Ionized Gases, 2001, July 17-22, Nagoya, Japan, Vol.3, pp.293295.

[4]. Handbook of Vacuum Arc Science and Technology, Eds. R.L. Boxman, D.M. Sanders, and P.J. Martin. NP, New Jersey, USA, 1990.

[5]. NIST Atomic Spectra Database Lines Data, http://physics.nist.gov

[6]. Vayner, B., Galofaro, J., and Ferguson, D. $33^{\text {rd }}$ AIAA Plasmadynamics and Lasers Conference, May 20-23, 2002, Maui, Hawaii (AIAA Paper 2002-2244)

[7]. Vayner, B., Galofaro, J., Ferguson, D., and Degroot, W. $40^{\text {th }}$ AIAA Aerospace Sciences Meeting\&Exhibit, Jan.14-17, 2002, Reno, NV (AIAA Paper 2002-0631). 